\title{
Design of Printing System of Forest Right Certifications
}

\author{
Weili Kou $^{1, a}$, Zhe Luo *, 2, b, Hua Yuan ${ }^{3, c}$, Tangsong Zhang ${ }^{3, d}$ \\ ${ }^{1}$ School of Computer and Information, Southwest Forestry University, Kunming, Yunnan 650224, \\ China \\ ${ }^{2}$ Business School of Finance and Economics, Yunnan College of Business Management, Kunming, \\ Yunnan, 650106, China \\ ${ }^{3}$ School of Forestry, Southwest Forestry University, Kunming, Yunnan 650224, China \\ akwl_eric@163.com, bufokunming@qq.com, 'stephenyuan@163.com, ${ }^{\mathrm{d}}$ ztangsong@126.com
}

Keywords: forest right system reform, printing system, forest right certifications

\begin{abstract}
The forest right certification is an important result of forest right system reform in China. The forest right certification printing is a very important work of collective forest right system reform. However, the traditional printing method of forest right certifications is tedious, detailed, and time-consuming. A printing system model is presented for printing of forest right certifications. System architecture with six layers, printing algorithms, key technologies, and methods are designed in this study. Dynamic model strategy is taken to enhance system flexibility. Memory cache and two-level querying techniques are employed to optimize books and pages splitting. The model was implemented and applied in more than 20 Counties of China, and the printing effects are more clearance, normalization, and readable.
\end{abstract}

\section{Introduction}

To promote forestry development on the premise of ecosystem construction and innovate in institutional mechanisms for collective forest management, collective forest right system reform has been implemented in China. Forest right certification printing is a very important work of collective forest right system reform, which is detailed, time-consuming, and heavy workloads [1, 2]. Traditionally, printing work is manually done, and is tedious, inefficient, costly, and time-consuming. Some information systems of forest right system reform are developed by researchers in the field of forestry ${ }^{[3-5]}$. So an efficient way of forest right certification printing is on urgent demands. Computers with high computing speeds, accuracy, and intelligence are extensively used to improve work efficiency in variety of fields. Information technology can help to automatically do works that can be abstracted as a certain process. Printings of forest right certifications is such a work, therefore, this study tries to enhance work efficiency based informatization technology.

\section{Background and Related Work}

Forestry work efficiency always was enhanced through modern information and computer technologies based on automation of work processes. Plenty of forestry software and systems are developed to support various special work in the filed of forestry. Due to different application environments and work requirements, special information models are needed to fit to the special work. Although printing of forest right certifications is similar with previous forest information systems in some common modules, it has some special characteristics. For instance, printing of forest right certifications is related to various data types, such as basic land attributes, attached figures, and documents. 


\section{Development Methods}

Prototyping method. Prototyping method is used to develop the printing system of forest right certifications. Firstly, prototyping system was developed by structure analysis designs and object oriented technologies based on initial requirements of users. Secondly, the prototyping system is tried and evaluated by users, and some suggestions and advice are collected for accurate defining the printing system requirements of forest right certifications. Thirdly, According to the suggestions and advices collected in step 2, an improving version is issued for users to use and evaluate. Fourthly, the step 2 and 3 are iterated until the user needs are met. Fifthly, a final version of the system is developed and issued to all users.

Object oriented method. Object oriented method with characteristics of stability, reusability, and maintainability can quickly and efficiently iterate prototyping method ${ }^{[6]}$. Object oriented method was used in the work of requirement analysis, system designs, and testing through the whole development work. Several classes are designed in this system, such as DataAccess, LandPatches, PrintingInformation, and Certifications. Meanwhile, object-oriented methods and events are used in the development of printing system of forest right certifications.

\section{Design of Printing System of Forest Right Certifications}

System Requirements Analysis. The system should meet the basic application needs to conventiently register initital forest right information, quickly display updated certfication printing information, and subsentially enhance certification efficiency. It should support users to conviently edit attached figures and attribute data, release and deploy new versions, ensure system security, manage users and roles, dynamically provide aboundant statistics reports. The system assigns different roles to different users to ensure system security ${ }^{[7]}$. The printing status of every land patch should be easily queried and listed by various users.

System Architecture. The system architecture consists of six basic layers, includes physical, software, data, data accessing, model, and interface layers from bottom to top (Fig. 1). Physical layer consists of computer hardware and network devices, including CPU, memory, .hard disks, printers, scanners, and other devices. The software layer includes operating systems (such as Windows, Linux, and Mac iOS) and relational database management systems (such as Microsoft Access, SQL Server, and Oracle). Data layer consists of attached drawings, the printing model of forest right certifications, forest right certifications, forest lands, and auxiliary data. Data accessing layer takes two objects to implement data access. It employs File System Object (FSO) to access document type data, ActiveX Data Objects (ADO) to access structured data. Model layer is designed for management of models used to implement system functionalities, includes encryptions and authentications, forest right statistics, forest querying, roles management, data accessing, page setup of forest right certifications, system initializations, generation of forest right certifications, previews of forest right certifications, printing of forest right certifications, certification number setup, and other models. Interface layer provides various interfaces of system maintenance, ordinary users, ordinary administrators, and super administrators. Indices are able to celebrate querying speeds of databases. For improve data accessing efficiency, indices are created on some fields of tables.

\section{The Printing Algorithm of Forest Right Certifications}

A printing algorithm was designed to print forest right certifications (Fig. 2). This algorithm is different from traditional printing system in two aspects. (1) The algorithm takes a dynamic model strategy to enhance system flexibility. We used models to predefine different forest right certification formats, and store them into a model database. The system automatically and dynamically chooses a suitable model according to different requests from users. (2) Fast books and pages splitting method was presented based on memory cache and two-level querying technology. Books and pages splitting information are small and cached in memories to give users a rapid 
response. The algorithm takes a novel strategy with dynamic fetching mechanism to manage detailed information with relative big volumes of every page.

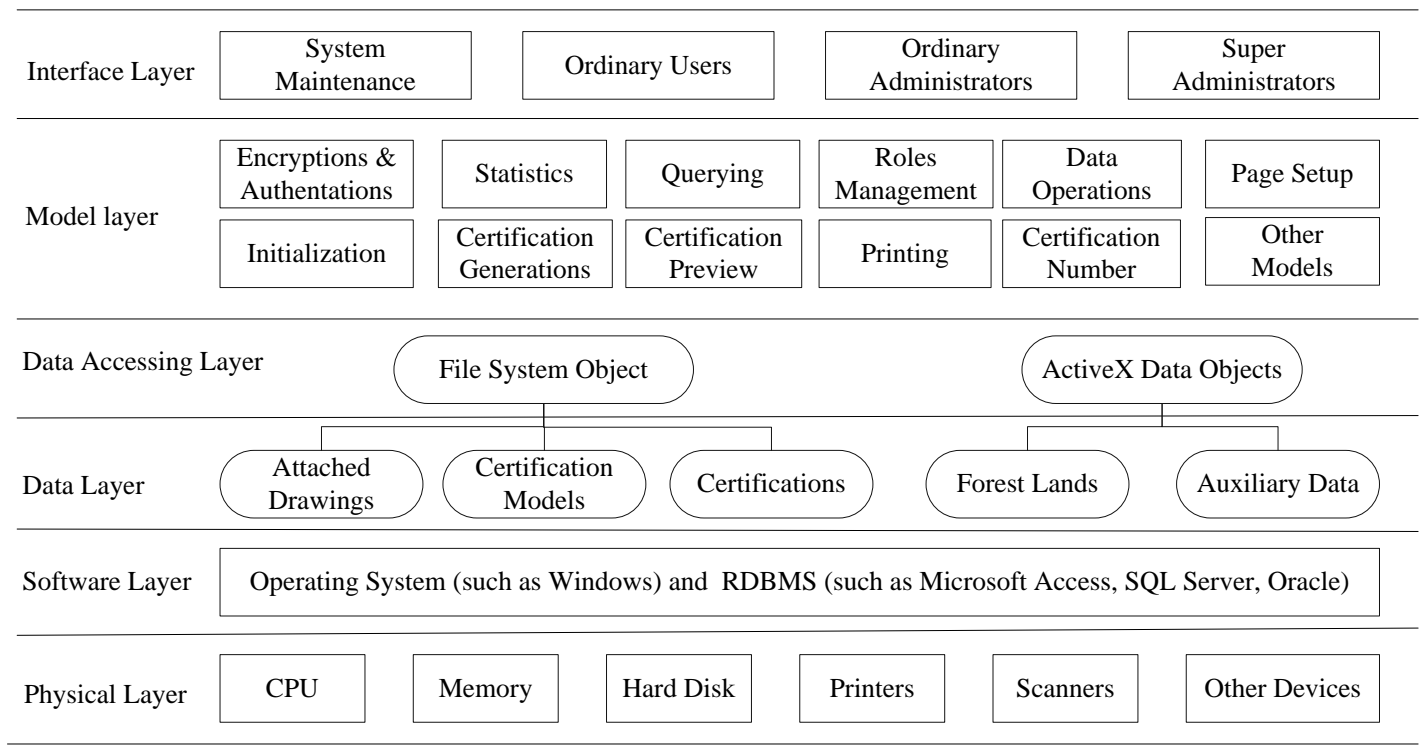

Fig. 1 The printing system architecture of forest right certifications

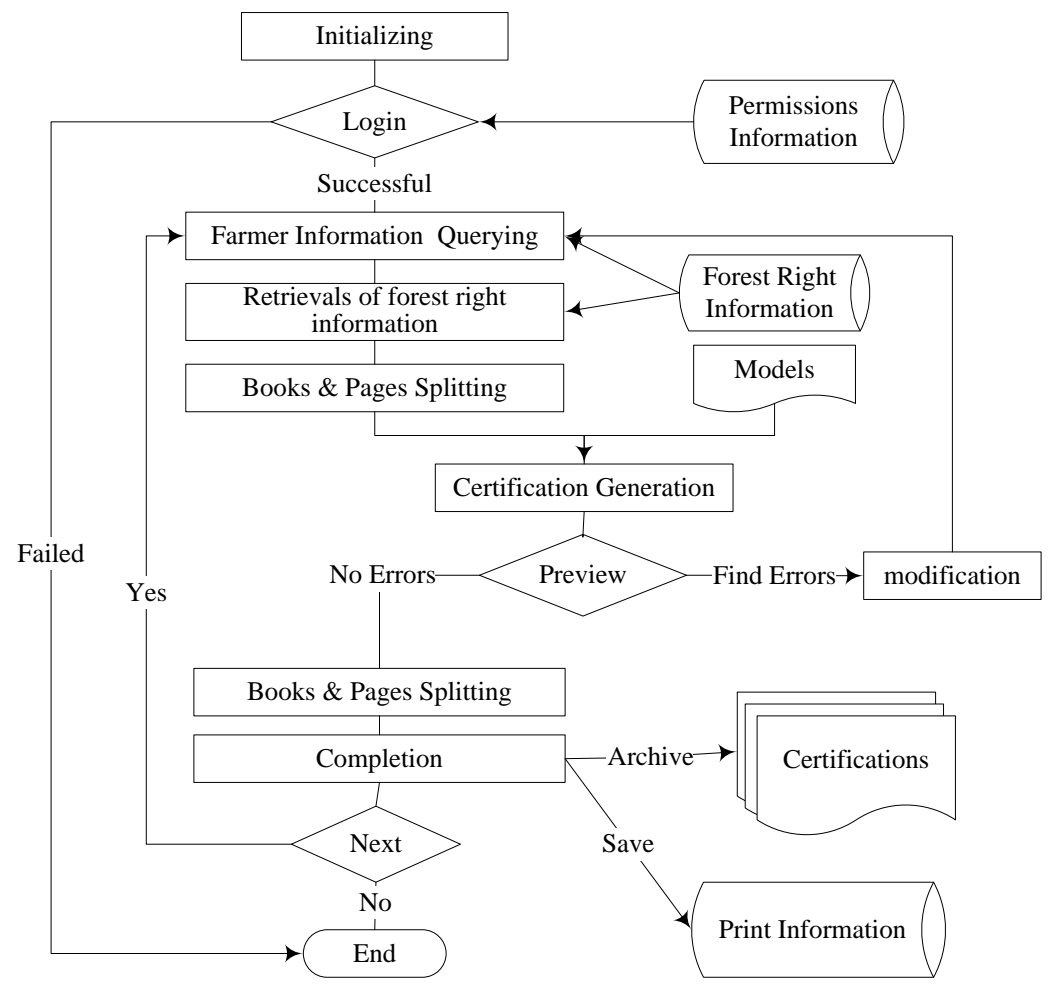

Fig. 2 The printing algorithm of printing system of forest right certifications

\section{Results}

A printing system of forest right certifications is implemented by Visual Basic programming language on Windows XP with Microsoft Office 2003 based on the model, which has been used in more than 20 Counties of China to help improving printing efficiency. The printing speed is ten-times higher than traditional methods, and the printing effects are more clearance, normalization, and readable. Additionally, the friendly user interfaces are able to help users conveniently completing their work. 


\section{Conclusions}

A printing information framework of forest right certifications is presented and implemented to enhance work efficiency. The study promotes the development of forestry informatization by providing a generalization printing information model of forest right certifications. The application results of the model performs well, the model is reasonable, flexible, and easy-to-use. In future study, printing information model under Internet environment will be designed and presented to facilitate data sharing. Additionally, parallel computing paradigm will be used to improve data processing efficiency of forest right certifications.

\section{Acknowledgement}

This work is supported by the Supported by Research Center of Kunming Forestry Information Engineering Technology, the Yunnan Provincial Research Foundation for Basic Application Research, China (No. 2011FB070), the Science Research Fund of Yunnan Provincial Education Department, China (No. 2014Y324), the Natural Science Research Foundation of Education Bureau of Yunnan Province, China (No. 2011Y282), , and Teaching Research Foundation of Southwest Forestry University (No. YB201120).

\section{References}

[1] Xing-bin Sun, Fei Wang, Huitin Yan. Distribution Based Design and Implementation of Forestry Rights Certificate - Management Information System in Hunan Province[J]. Journal of Northwest Forestry University. Vol. 28 (2013): p. 230

[2] Zhongjian Deng, Rulian Zhou, Xiao-yan Wei. Making System of Attached Figures of Forest Property Right Certificate Based on Arcgis Engine[J], Forest Inventory and Planning, Vol.33 (2008):p. 21

[3] Jnnnan SHI, Zhihao Long. Research on Authorized Area based Access Control Technique[J], Computer Knowledge and Technology, Vo1. 5 (2009): p. 10180

[4] Weili KOU, Hua Yuan, Tangsong Zhang. Research and Design of Management Information System of Forest Property Right Certification Based Oil GIS[J], Vol.34 (2009): p. 77

[5] Fang Wang, Yun Li. Implementation of Forest Right Certification Approval Process-control Subsystem witll JBPM[J], Agriculture Network Information, Vol. ( 2011): p. 31

[6] Xinqiu DAN, Nanfei Wu. On Design of Forest Ecological Tourisin System[J], Central South Forest Inventory and Planning., Vol. 20 (2001): p. 48

[7] Qiongyu Cai, Chungan Li, Ya Liu. The Overall Design for Management Information System of Forest Ownership Certification[J], Central South Forest Inventory and Planning, Vol. 30 (2011): p. 31 